\title{
Developing lifelong learners: a novel online problem-based ultrasonography subject
}

\author{
Laura C. Minasian-Batmanian,* Anthony J. Koppi** and Elaine J. Pearson*** \\ *Department of Biomedical Sciences, Faculty of Health Sciences, \\ University of Sydney \\ **Educational Development and Technology Centre, University of South Wales \\ ***School of Computing and Mathematics, University of Teesside \\ email: I.batmanian@cchs.usyd.edu.au
}

Online learning environments have a major role in providing lifelong learning opportunities. Lifelong learning is critical for successful participation in today's competitive work environment. This paper describes an online problem-based learning approach to the creation of a student-centred learning environment for the study of the biological sciences subject in the Graduate Diploma of Applied Science (Medical Ultrasonography) course at the University of Sydney. The environment is interactive and collaborative, with all communication taking place online. Students work in groups to study clinically relevant problems. $A$ Web-database system provides learner control in the process of knowledge acquisition, access to reference materials on the Internet and communication with the tutor and with peers through synchronous chat and asynchronous threaded discussion forums. Other online features include a protocol for problem-solving, self-assessment and feedback opportunities, detailed help, streaming audio and video and pre-course, ongoing and post-course questionnaires. This technology may be adapted to a range of disciplines and can also be utilized in on-campus teaching.

\section{Introduction}

There have been significant advances in the practice of sonography since the introduction of the University of Sydney course in 1991. The entire knowledge base can no longer be taught within the constraints of time allotted to the subject. Moreover, encouraging students to be self-directed, independent learners is more important than ever, since the field of sonography has reached a stage where there is an urgent need for new skills.

In addition, there has been a range of social and economic factors that have resulted in major changes in higher education and have contributed to the current educational climate 
(Campion, 1995; Edwards, 1995). These changes have challenged universities to introduce teaching and learning strategies that cater for different client groups using forms of delivery that increase access to learning opportunities. In particular, there are demands for the university sector to provide for a larger and more diverse cross-section of the population, to cater for emerging patterns of educational involvement which facilitate lifelong learning, and to include technology-based practices in the curriculum (Renner, 1995).

These developments imply a student-centred approach to learning. Given the current economic climate in higher education and the need to maintain pedagogically sound practices, there is the need to consider learning within a developmental framework (Laurilland, 1993) which has lifelong learning as a major outcome. The introduction of problem-based learning (PBL) in the biological sciences subject of the Graduate Diploma of Health Science (Medical Sonography) at the University of Sydney in 1996 allowed students to be more independent with greater self-direction in their study programme. The subject content and structure have changed to make them relevant to the needs of sonographers in a changing professional environment. Online delivery is seen as a way of providing student-centred lifelong learning opportunities. The issue for those designing these courses is how to provide more appropriate and flexible educational opportunities for the learner, given that the importance of knowledge acquisition is the primary focus of the educational process.

This paper describes how technology was incorporated into the PBL approach in 1998 to offer enhanced learning capabilities, through the development of an innovative programme delivered entirely in distance learning mode. The online programme ran for the first time in 1999. It has attracted local, rural, interstate and New Zealand students. This accessibility has increased the size of the market, as well as making the offering of courses overseas viable. At the same time it has accommodated the time limitations of the mature-age parttime professionals of this course, without the need for simultaneous traditional university training.

\section{Design}

The multidisciplinary in-house team involved in the development of this programme over a period of six months spent 700 hours in the developmental stage of this programme at a cost of $\$ A 22,400$. The team consisted of an academic, the academic developer from the Centre for Teaching and Learning, an educational technologist and a Web developer. They had the task of collectively providing a comprehensive view of specific subject knowledge, and a pedagogical awareness of the principles underlying optimal student learning and technical skills in Web design and development. The team was conscious that the learner, not the technology, is central to the design, which, in turn, is based on sound educational principles (Godfrey, 1996). Standard Internet technology, MS SQL Server Database and Cold Fusion software were used.

The team sought to embed the fostering of generic attributes and lifelong learning skills in the program. As McAllister (1997) points out, the graduates of today are expected to be 'beginning practitioners' who become 'critical consumers of information and lifelong learners who maintain competence in their discipline, expand and test their own knowledge 
and skills, and contribute to the expansion of knowledge in the field'. The goals of vocationally-based education have changed from focusing on training and instruction to include an emphasis on the development of generic attributes essential to professional competence and lifelong learning (Bates, 1997; Higher Education Council, 1992; Kirkwood, 1998; Ramsden, 1992; Savery and Duffy, 1994). Engel (1995) summarized these as comprising the acquisition of:

- discipline-specific knowledge and competencies;

- generally applicable competencies, for example, ability to adapt to change and participate in change, communicate, collaborate in groups, and be self-directed lifelong learners who can apply critical reasoning and a scientific approach to decision-making in unfamiliar situations.

In order to achieve these aims, designing and redesigning content and methodology for online delivery was important, as was reconsidering previously accepted and often unquestioned practices.

The social aspect of learning present in traditional university-based education is often missing, neglected or poorly considered. Online courses often rely on simply taking written text or lecture notes and presenting them digitally. Students are expected to learn independently by reference to the written or presentation materials the lecturer would normally deliver in a classroom situation. This site, however, takes advantage of multimedia facilities to provide added value to distance learning. It combines the independence of distance learning with the social aspects of university-based learning. The site therefore contains no lecture notes, but simply presents a text description of twelve case studies that the students are required to engage with. Students work in groups of six, through the online problem identification process (Protocol) to study the pathophysiological knowledge necessary to understand and solve these cases.

The biological sciences online environment has been designed for maximum flexibility. It allows students from a variety of backgrounds to construct a study plan according to their interests, expertise and availability. An individual can opt to be delegated to research certain objectives generated by the cases. Those with particular specialties are placed together to act as resource people for the whole online community. An online calendar was incorporated to assist students in scheduling their discussions. The flexible learning approach offers the student increased choice in what to learn (students devise their own objectives); how it is learned (the learning style they choose to follow from the resources supplied); and when and where learning happens (whether it is in a face-to-face situation, at the end of a telephone line as in teleconferencing, or online conferencing).

When designing the flexible learning environment, care was taken to develop domainspecific educational strategies. These include explanatory notes in the form of text/audio hints (Figure 1) and streaming video (Figure 2), a flow chart to explain the step-by-step Protocol (Figure 3), exercises in the form of self-evaluative tests and a diagnostic exam, resources, assessment requirements and group discussion facility via chatspace and discussion forum.

The visual elements are carefully planned to take account of non-technical users. The visual and navigational interface is designed for coherence, accessibility and clarity. The 


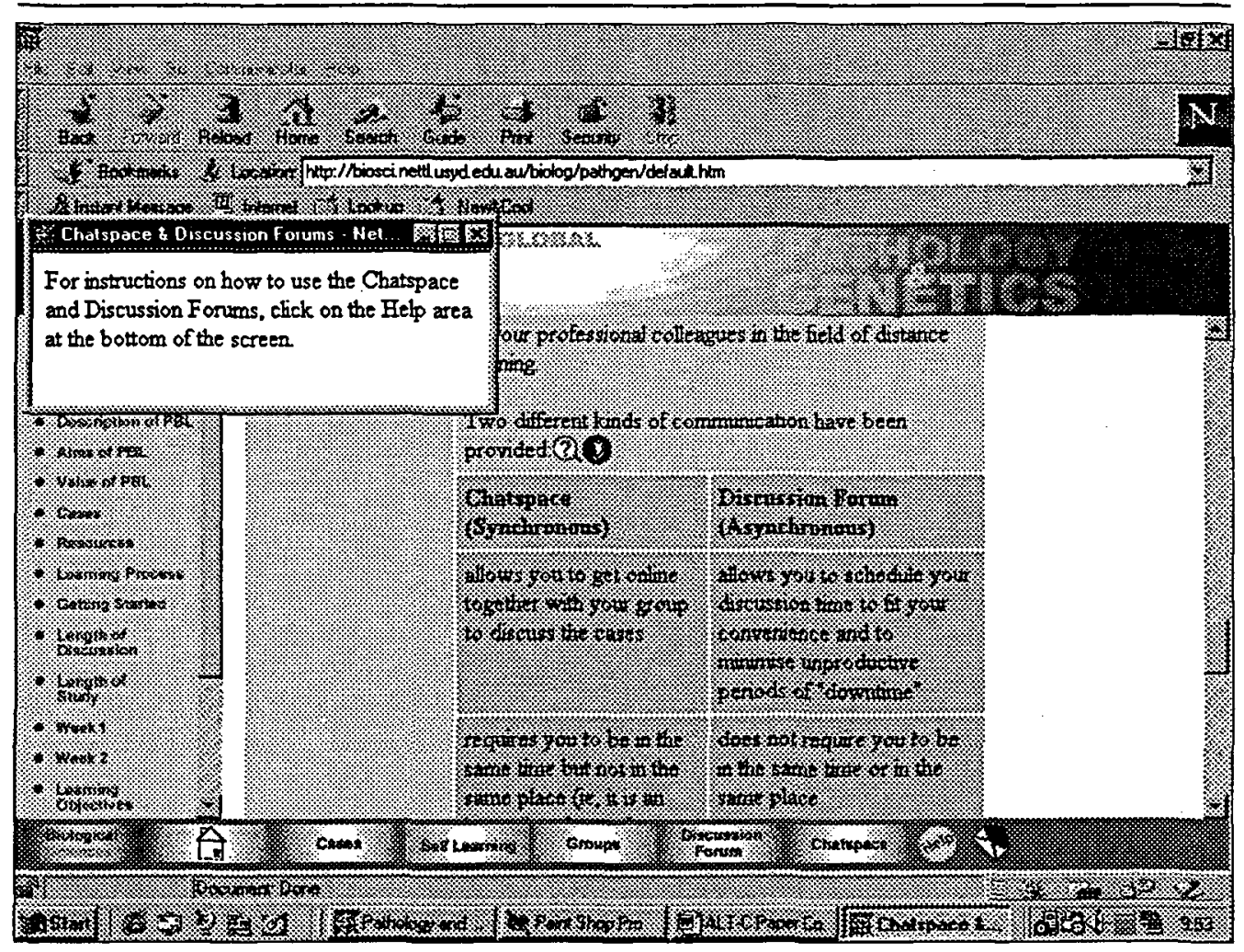

Figure 1:The pathology/genetics screen showing the use of audio hints

interface is clear with clean, crisp graphics and a subtle use of colours. The design utilizes plenty of white space, which gives a coherent feel to the display and clearly differentiates the navigational and display elements. The graphics give the impression of ultrasound without making the medical emphasis of the site too obvious. The menu structure makes the site very easy to navigate with the navigation bars remaining constant throughout. Information is arranged in three different, colour-coded sections in the site, for easy identification: pathology/genetics (blue), self learning (purple) and groups (green).

The explanation to the students of the teaching and learning methods employed is clear and comprehensive and avoids the use of jargon. Assessment is clearly explained and the course provides a spread of relevant assessment. The chat space and discussion forum provides students with the opportunity for collaborative learning online and self-tests assist students in monitoring their own progress. A series of evaluation questions provide feedback to the facilitator on most aspects of the subject and its delivery.

Self-directed learning is a feature of both traditional distance learning and university-based courses, but in distance learning it is sometimes almost the only pedagogic approach taken. Online learning can take advantage of multimedia to make a wide store of information available at the click of a button, giving all students equality of access. The use of hypertext with direct and associative links gives flexibility to the students' approach to learning. It helps to orientate the student to independence and self-responsibility in learning, a basis for 


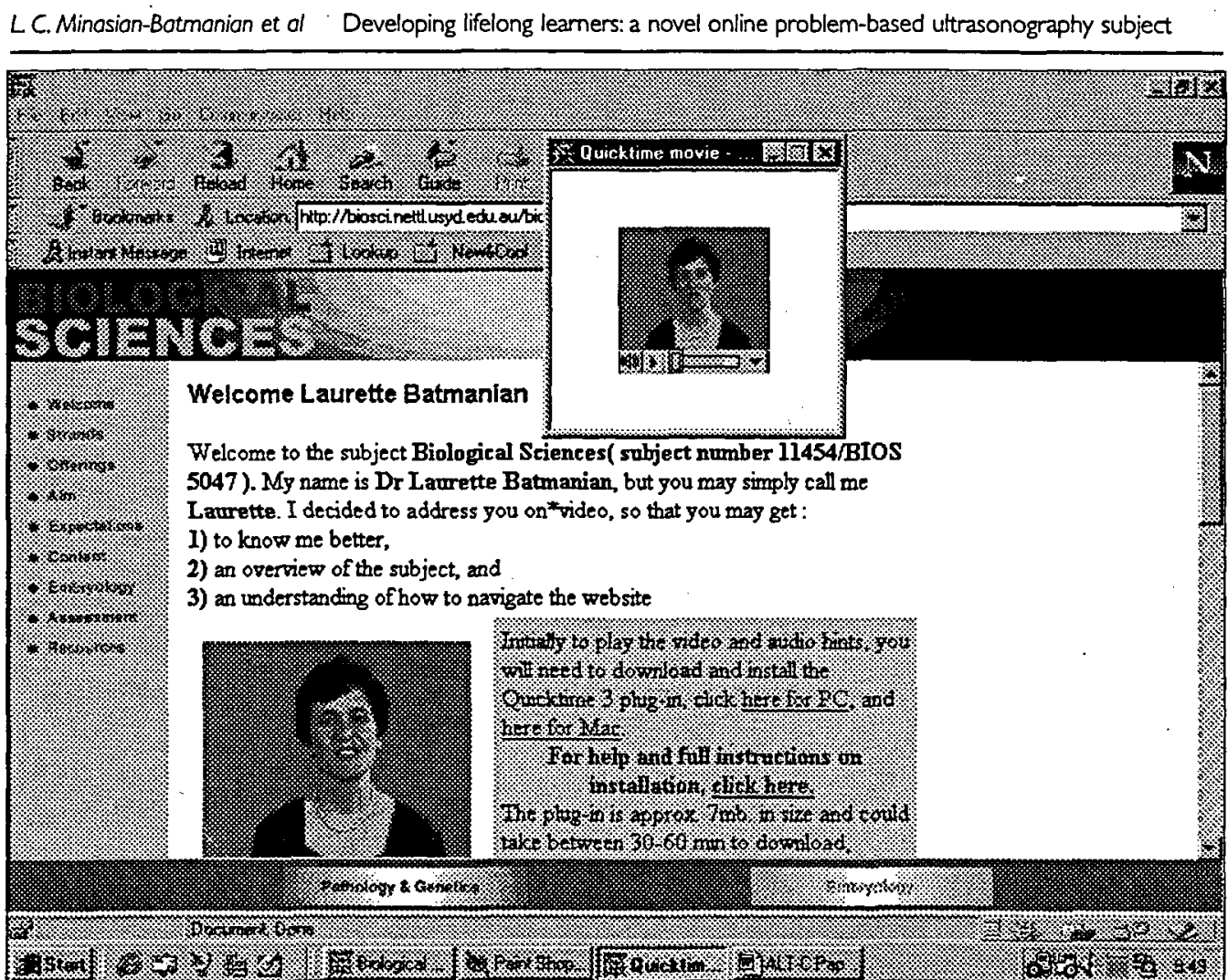

Figure 2:The biological science screen showing a streaming video

acquiring lifelong learning habits (Koppi, Lublin and Chaloupka, 1997). This site allows browsing, searching and guided discovery. The student can browse the extensive links to bibliographical references, other sites on the Internet or video clips, starting wherever they wish, following a chain of linked information intensively or skipping from one resource to another following associative links. Alternatively the student can make detailed searches using the same resources for specific information. There are also links from key words or phrases to specific and detailed information on particular aspects within the site.

The advantage of flexible delivery is that it includes learner control over content, time, place and method of learning. Online course materials are specially designed for studying when and where the students choose. The students can phone, fax, mail, or email teaching staff whenever they need help. Frequently asked questions regarding the educational aspects of the site are answered online. A suggested timetable (Figure 4), recommended length of discussions and a study schedule have been included to give participants an estimate of the time involved in this subject and the rate at which they can expect to progress. To engage students in the learning process, a case Proforma was incorporated, which summarizes the steps in the Protocol to guide the students through the learning process. Although a comprehensive online help system is provided, a fifty-page User Site Guide is mailed to students before semester starts, to familiarize them with the learning environment and navigational features. 


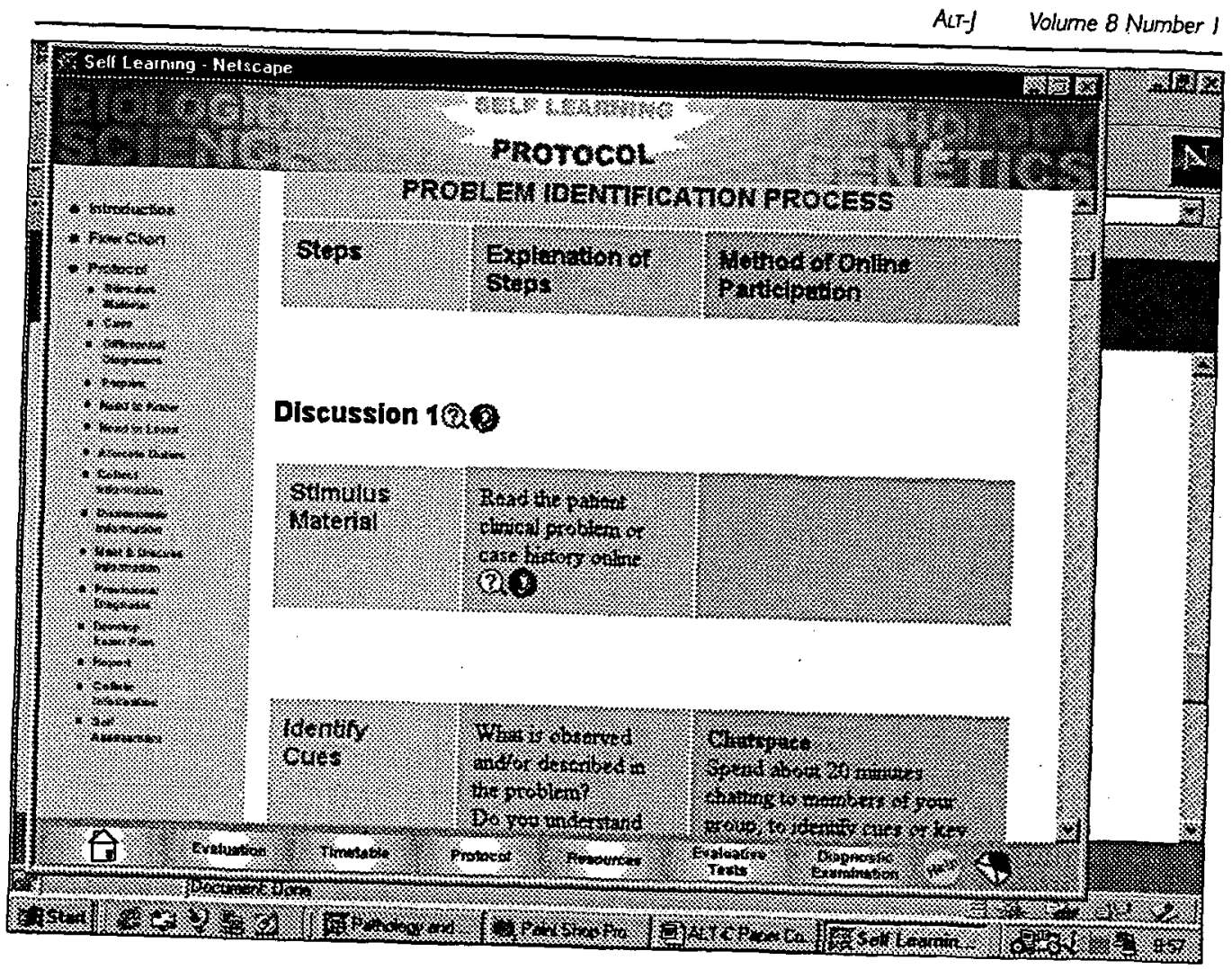

Figure 3:The Protocol flow chart

\section{Teaching and learning methodology}

Students are made aware from the outset of the teaching and learning methods adopted and are given detailed guidance on the philosophy, the features of the approach and the learning outcomes. This guidance provides early scaffolding to the students and immediately invites them to take ownership of their learning. This scaffolding is reinforced by the use of audio and video to introduce the facilitator to the student.

The entire knowledge base cannot be taught within the constraints of time allotted to the subject. Therefore, twelve cases were specifically chosen to cover the most commonly seen problems in ultrasonography practice, or those that illustrate significant principles particularly well. Clinical problems, the most common form of request presented in private practice and case histories, used to simulate the information provided in hospitals, have been incorporated. Problems were designed to begin with simple examples, becoming increasingly complex so that students can draw on previous knowledge.

\section{Collaboration}

Collaborative learning is a concept that is generally missing from traditional distancelearning courses. A particular feature of this site is the emphasis on group activity - indeed part of the assessment is based on the students' contribution to group work. Student 


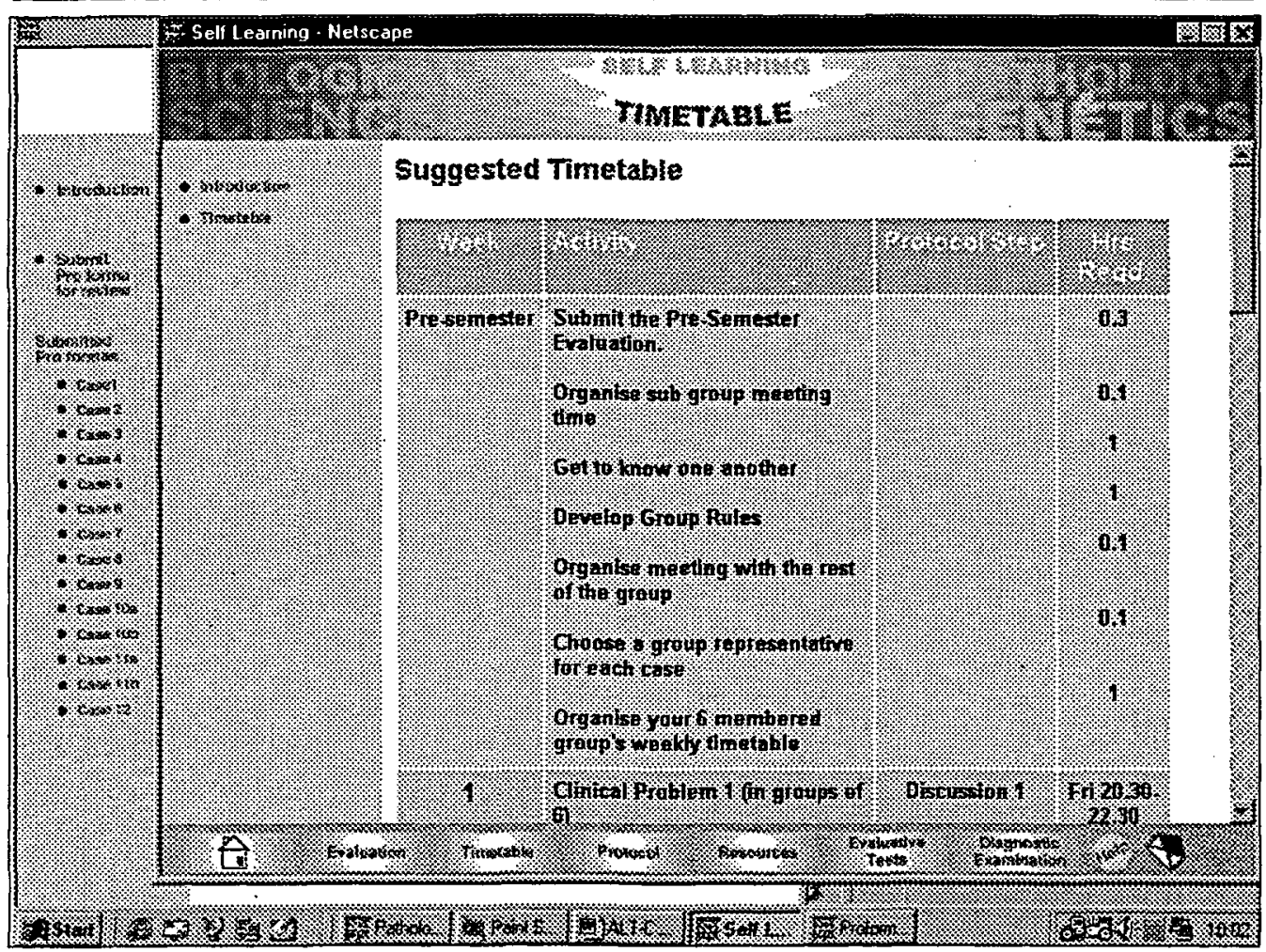

Figure 4: Screen showing the suggested timetable.

success is assessed in terms of the student's ability to solve problems, communicate ideas, present information and learn how to learn, rather than simply to repeat facts. The site includes discussion groups, forums, a chat space and facilitator/student communication:

- Discussion groups are designed for small groups of students to meet online and discuss the case studies and to identify and work through the problems together. The small groups are designed to create coherent units and a group identity. This means that individuals are less likely to drop out of discussions or 'lurk' and fail to participate. Students are given advice on the structure, behaviour and timing of group discussions. Forums are designed to provide whole group discussion and interaction - an online replacement for the university-based seminar.

- There are three types of forum: (i) case studies for wider discussion of the problem, (ii) feedback where the students can discuss feedback given by the facilitator, and (iii) general discussion where students are able to raise any matters not necessarily directly related to the subject content.

- The chat space provides an opportunity for students to talk in real time (anonymously if they prefer) and to post their own opinions, references and resource links.

- Email, fax and phone communication with the facilitator are provided for support, feedback and assessment. 


\section{Feedback}

In this online course, the lecturer becomes a facilitator of information (electronic, verbal, etc.) by guiding, advising, commenting on progress and offering explanations, or adopting the role of co-learner. Students are encouraged to submit alternative information with which they may be familiar from their work situation or through their literature or Web searches. This encourages the sense of interdependence and validates the knowledge that students bring to the learning situation.

The online learning environment provides the facilitator with a greater level of awareness on the progress of individuals. In the biological sciences subject we tried to engage the firstyear students in a number of ways. Thirty-six students were enrolled in the subject. They were given an optional face-to-face introductory meeting with academic staff before the start of the course. Twelve students decided to learn in a face-to-face situation, twelve by online conferencing alone and twelve using a combination of the two processes. The facilitator was scheduled to attend the first four weeks of synchronous chat discussion forums, to assist students using the online environment with resolving immediate problems concerning the Protocol.

The students were initially encouraged to interact with three students of the group of six to get to know one another before semester started. They exchanged information about interests and hobbies on a personal level; their specialty at a professional level; and any other issues of common interest that they would like to share with the other students and the facilitator. Online participant profiles, which are accessible and amenable to change, were provided to personalize the design of the site (Pillay, Boles and Raj, 1998). This extracurricular activity was used as a focus to acquaint the students with their peers and to introduce them to the new learning environment. This was found to be beneficial before students worked in groups on their clinical problems or case studies. The groups were provided with guidance to help them devise criteria for working effectively as a team. This reflects the skills they will require in their future professional and social lives. The proportions of both peer and facilitator support have been increased to four hours per week from the normal rate of two hours per week. This is intended to overcome feelings of isolation and to allow for the fact that learning and motivation are significantly improved for distance-learning participants by increased communication and feedback. The facilitator was always on duty to respond to email, fax or phone, although in the future this will be restricted to certain hours in the week.

\section{Assessment}

The online subject features both formative and summative assessment. Formative assessment is provided through a series of self-evaluative tests at various stages in the course and a diagnostic mock examination. Self-tests enable the students to monitor their own progress in a non-threatening situation and thereby take control of their own learning. The use of formative assessment through questionnaires or short-answer questions means students are able to test their own ideas against 'expert' opinions. These tests are also useful in assisting students in the identification of learning goals and areas of difficulty and provide a means of reflecting on their on-going progress. Other formative assessment comes from peer group discussion and feedback from the facilitator. 
Summative assessment emphasizes the importance of collaborative learning, with a proportion of the marks based on contribution to group work ( 5 per cent), as well as ten reports submitted by the group ( 40 per cent). Individual assessment ( 28 per cent) is based on an end examination comprising multiple-choice and short essay questions. Students are provided with additional scaffolding in the first clinical problem, which is presented as a worked example and hints are provided to help solve case history two, giving an example of the scope, focus and level of work required for the assessed cases.

\section{Evaluation}

Staff at the University of Teesside, UK, evaluated the biological sciences subject. The evaluation was based on an examination of the features that contribute to the PBL approach as well as visual, content and navigational design elements. After evaluation, the subject was presented to the students as an example of best practice in online learning. The results of the evaluation have been incorporated into this paper.

This online environment enables integration and co-ordination of theoretical and clinical material, as well as encouraging the development of skills in logical reasoning, critical thinking, communication and self-directed learning (Grabinger, Dunlap and Duffield, 1997). Biggs (1989) found that teaching that gave evidence of deep learning contained one or more of the following: an appropriate motivational context; a high degree of learner activity; interaction with others, both peers and teachers; and a well structured knowledge base. The students were highly motivated through the direct link between the cases and the demands of the subject and its assessment. There was a high degree of learner activity with brainstorming, role-play and feedback (Moore, 1993). Students had to interact constantly with their peers and facilitators. We believe that the programme described in this paper has led to deep learning, as evidenced by their results of 94 per cent graded passes this year, as compared with 84 per cent last year. Although evaluation of student responses is yet to be fully analysed, preliminary feedback has been most favourable. Some representative comments included:

'The best aspect of the subject was the problem-based learning approach.'

'The tie-together of pathology and its effect on the patient and what we should expect to observe with ultrasound made the subject relevant.'

'Diagnosing the case studies was interesting. It was good to be in groups as we got to know other people in more depth.'

'Links were very helpful even now after bioscience has finished.'

'My skills in written communication as well as the use of technology was greatly improved.'

\section{Discussion}

Consistent with the philosophy of critical and reflective practice, we have attempted to accommodate the attributes of lifelong learning and critical reasoning skills development, described by Candy (1994), to the study of ultrasonography. The cases require that the students: 
- ask questions of the facilitator and of each other to determine objectives, undertake critical appraisal of the relevant literature, and self-evaluation of understanding;

- are aware of the interrelationships between theoretical and applied knowledge and acquire an understanding of ultrasound procedural limitations;

- develop a knowledge of current resources, frame researchable questions, locate, evaluate, manage and use information in a range of contexts, retrieve information using a variety of media, including Web links, videos, literature searches; decode information in a variety of written and graphical forms; and critically evaluate information to be submitted in the report;

- are capable of working on their own, interact within a team, and develop self organizational skills as well as an appreciation for multiple perspectives;

- acquire a variety of learning skills, an awareness of their own personal strengths, weaknesses and preferred learning style; develop different strategies for learning according to context and understand the differences between surface and deep learning.

\section{Conclusion}

This paper has shown that technology can assist in the adoption of a more student-centred approach to teaching and learning in which students develop knowledge, skills and resources that can assist them to a stage of 'learning by discovery', through a process of discussion, discovery, interaction, adaptation and reflection. It has the advantages of providing increased accessibility, immediate feedback, interactive learning and a more flexible environment. It provides both peer and tutor support and guidance, as well as a wealth of resources. The subject encourages active learning, without leaving the student awash in a sea of information, by providing guidance on how to begin addressing the problems introduced in the case studies. It provides a complete environment from introduction to assessment and evaluation.

Another advantage of this site for the courseware developer is that the format adopted is easily transferable to a different subject and course, making reuse a practical proposition and thereby cutting down on future development. The template is currently being adapted to accommodate for the design and development of a postgraduate subject in Management in the School of Health Information Management, Faculty of Health Sciences, University of Sydney. Moreover, active interest has been expressed for use in undergraduate courses run by the Department of Biological Sciences and continuing education courses run by the Department of Obstetrics and Gynaecology, at the University. The site has been adopted by the University of Teesside as an example of good practice in teaching courseware design at masters degree level.

\section{Acknowledgements}

Laura Batmanian wishes to thank Steve Clark and Andrew Lovell-Simons for their assistance in designing and developing the site; Jason Bayly for Web design, programming and co-ordination; and Angus Denton for the productions of the streaming video and audio hints. 


\section{References}

Bates, A. (1997), 'The impact of technological change on open and distance learning', Distance Education, 18 (1), 93-109.

Biggs, J. (1989), 'Approaches to the enhancement of student learning', Higher Education Research and Development, 8 (1), 7-25.

Campion, M. (1995), 'The supposed demise of bureaucracy: implications for distance education and open learning - more on the post-Fordism debate', Distance Education, 16 (2), 192-216.

Candy, P. (1994), 'Developing lifelong learners through undergraduate education', Commissioned Report No. 28, National Board of Employment, Education and Training, Canberra: Australian Government Printing Service.

Edwards, R. (1995), 'Different discourses, discourses of difference: globalisation, distance education and open learning', Distance Education, 16 (2), 241-55.

Engel, C. E. (1995), 'Medical education in the twenty-first century: the need for a capability approach', Capability, 1 (4), 23-30.

Godfrey, R. (1996), 'The World Wide Web: a replacement, displacement, supplement or adjunct of traditional methods', in James, C. P. and Vaughan, B. (eds.), ASCILITE 96: Making New Connections, Proceedings of the 13th Annual Conference of the Australian Society of Computers in Learning in Tertiary Education, University of South Australia, Adelaide, 221-34.

Grabinger, S., Dunlap, J. and Duffield, J. (1997), 'Rich environments for active learning in action: problem-based learning' $A L T-J, 5$ (2), 5-18.

Higher Education Council (1992), 'Higher education: achieving quality', AGPS, Canberra, $20-2$.

Kirkwood, A. (1998), 'New media mania: can information and communication technologies enhance the quality of open and distance learning?', Distance Education, 19 (2), 228-41.

Koppi, A. J., Lublin, J. R. and Chaloupka, M. J. (1997), 'Effective teaching and learning in a high-tech environment', IETI, 34, 245-51.

Laurilland, D. (1993), Rethinking University Teaching: A Framework for the Effective Use of Educational Technology, London: Routledge.

McAllister, L. (1997), 'Towards a philosophy for clinical education', in McAllister, L., Lincoln, M., McLeod, S. and Maloney, D. (eds.), Facilitating Learning in Clinical Settings, Cheltenham: Stanley Thornes, 214-45.

Moore, M. (1993), 'Three types of interaction', in Harry, K., John, M. and Keegan, D. (eds.), Distance Education: New Perspectives, London: Routledge, 19-24.

Pillay, H., Boles, W. and Raj, L. (1998), 'Personalising the design of computer-based instruction to enhance learning', $A L T-J, 6,17-32$. 
Ramsden, P. (1992), Learning to Teach in Higher Education, London: Routledge.

Renner, W. (1995), 'Post-Fordist visions and technological solutions: educational technology and the labour process', Distance Education, 16 (2), 284-301.

Savery, J. R. and Duffy, T. M. (1994), 'Problem based learning: an instructional model and its constructivist framework', Educational Technology, 8, 31-8. 